\title{
Distributed stabilization control of rigid formations with prescribed orientation
}

\author{
Zhiyong Sun ${ }^{\mathrm{a}}$, Myoung-Chul Park ${ }^{\mathrm{b}}$, Brian D. O. Anderson ${ }^{\mathrm{a}}$, Hyo-Sung Ahn ${ }^{\mathrm{b}}$ \\ ${ }^{a}$ National ICT Australia and Research School of Engineering, Australian National University, Canberra ACT 0200, Australia \\ ${ }^{\mathrm{b}}$ School of Mechatronics, Gwangju Institute of Science and Technology, Gwangju, Republic of Korea
}

\begin{abstract}
Most rigid formation controllers reported in the literature aim to only stabilize a rigid formation shape, while the formation orientation is not controlled. This paper studies the problem of controlling rigid formations with prescribed orientations in both 2-D and 3-D spaces. The proposed controllers involve the commonly-used gradient descent control for shape stabilization, and an additional term to control the directions of certain relative position vectors associated with certain chosen agents. In this control framework, we show the minimal number of agents which should have knowledge of a global coordinate system (2 agents for a 2-D rigid formation and 3 agents for a 3-D rigid formation), while all other agents do not require any global coordinate knowledge or any coordinate frame alignment to implement the proposed control. The exponential convergence to the desired rigid shape and formation orientation is also proved. Typical simulation examples are shown to support the analysis and performance of the proposed formation controllers.
\end{abstract}

Key words: Formation control; formation orientation; coordinate system, rigidity theory.

\section{Introduction}

\subsection{Background and motivation}

Formation control for a group of autonomous mobile agents has gained much attention due to its broad applications in many areas including both civil and military fields. A key problem in this domain that receives particular interest is how to stabilize and maintain a geometrical formation shape in a distributed manner. In the recent survey paper Oh, Park, and Ahn (2015), different types of formation control strategies are reviewed and compared, among which two most commonly-used approaches are

- the linear displacement-based approach: the desired formation is specified by a certain set of inter-agent

\footnotetext{
* The material in this paper was partially presented at the IEEE Multi-Conference on Systems and Control, September 21-23, 2015, Sydney, Australia (Sun and Anderson (2015)) and the 54th IEEE Conference on Decision and Control, December 15-18, 2015, Osaka, Japan (Park and Ahn (2015)).

Email addresses: zhiyong.sun@anu.edu.au (Zhiyong Sun), mcpark@gist.ac.kr (Myoung-Chul Park), brian.anderson@anu.edu.au (Brian D. O. Anderson), hyosung@gist.ac.kr (Hyo-Sung Ahn).
}

relative positions which means that the orientation of the final formation is implicitly fixed;

- the nonlinear distance-based approach: the desired formation is specified by a certain set of inter-agent distances, and the orientation of the target formation is not implicitly or explicitly defined.

For the first approach, all the agents must have their coordinate bases with the same orientation (while the origins may be different) such that the desired relative position vectors are well defined and controlled between agents (see e.g. Ren and Beard (2008); Xiao, Wang, Chen, and Gao (2009)). This means that all the agents should be equipped with compass to guarantee their coordinate orientation alignments, which may not be practical in e.g. compass-denied environment. The coordinate frame requirement was largely ignored in early works on formation control (as reviewed in Oh et al. (2015)). It is only in recent years that the importance of coordinate frame issue has been recognized in formation controller design and implementation. In the case that initially all the agents in the 2-D plane have different local coordinate frames, one needs to design a combined control establishing coordinate frame direction alignment and linear displacement-based formation stabilization to ensure the convergence of a target shape Oh and Ahn (2014b). Furthermore, it has also been 
shown in Meng, Anderson, and Hirche (2016) that the assumption that all the agents have coordinate systems with the same orientation may not be realistic in practice as small perturbations in their local coordinate systems will cause unexpected behaviors for the displacementbased formation system. Thus in practice, a coordinatefree formation control system is always favorable. In Aranda, López-Nicolás, Sagüés, and Zavlanos (2015), a coordinate-free formation control strategy was proposed by including a rotation matrix in the formation controller. The advantage of the coordinate-free property of the proposed formation controller in Aranda et al. (2015) is paid by the price that the relative position measurements from all other agents should be available to each individual agent, which implies that the coordinatefree formation control in Aranda et al. (2015) is not a distributed one. Recent efforts also show that the bearing-based approach is another promising strategy to achieve a desired formation Zhao and Zelazo (2016). We note that such an approach however still does not resolve the strict requirement of the global knowledge of coordinate frame orientation for individual agent.

All these disadvantages on the coordinate frame requirement can be avoided in the distance-based formation setup. This is because that in the distancebased setup any global coordinate system defining a common orientation for all individual agents' coordinate frames is not required, and each agent can use its local coordinate basis to achieve a rigid formation shape (we refer the readers to Fig. 3 in Oh et al. (2015) for a comparison of coordinate basis requirement for these two approaches). Rigid formation control has been discussed extensively in the literature, most of which has focused on the convergence analysis of formation shapes (see e.g. Krick, Broucke, and Francis (2009), Anderson and Helmke (2014), Cortés (2009), Dorfler and Francis (2010), Oh and Ahn (2011), Tian and Wa (2013), Cai and De Queiroz (2015)). Note that in many applications involving multi-agent coordination, a formation with both a desired shape and a particular orientation is required. However, for distance-based rigid formation control, the orientation of the final formation is not controlled and actually not well defined, ${ }^{1}$ which may limit the practical application of shape controllers

\footnotetext{
1 We need to distinguish different meanings of orientation in the context of formation control. By regarding a rigid formation as a rigid body, the formation orientation relates to the overall rigid formation. The orientation concept in e.g. Oh and Ahn (2014b); Montijano, Zhou, Schwager, and Sagues (2014) refers to the orientation of the local coordinate frame for each agent. We will distinguish different meanings by referring explicitly to either formation orientation or coordinate orientation. Another orientation concept refers to the definition of signed area for a closed curve formed by a formation shape with a specific ordering of all agents (e.g. a triangle with positive/negative area). This concept will not be used in this paper.
}

discussed in these previous works. In this paper, we aim to design distributed formation controllers to achieve a desired rigid formation with a prescribed formation orientation.

\subsection{Related work}

The stabilization control of rigid formations with desired orientation was discussed in Pais, Cao, and Leonard (2009) by using the tensegrity theory and a projected collinear structure. However, the approach still requires all the agents to have knowledge of the orientation of a common reference frame. The problem of stabilizing only the orientation of rigid objects subject to distance constraints was studied in Wang, Markdahl, and $\mathrm{Hu}(2011)$, Markdahl, Karayiannidis, Hu, and Kragic (2012), by assuming that the rigid shapes remain constant which are not stabilized. Thus, the approaches in Wang et al. (2011) and Markdahl et al. (2012) cannot be applied to solve the formation stabilization control task in question. In our previous paper Sun, Mou, Anderson, and Morse (2014) we showed a feasible approach to move or reorient a rigid formation to a desired orientation by introducing distance mismatches; however, such orientation control approach, which is a by-product of the mismatched formation control problem, indicates that the final formation is slightly distorted compared to the desired formation. Furthermore, the orientation control in Sun et al. (2014) also requires global information in terms of all other agents' positions which is contrary to the formation control task using a distributed approach.

In this paper we propose feasible and distributed controllers to achieve both rigid shape stabilization and formation orientation control with minimal knowledge of global coordinate orientation for the agent group. The basic idea underlying the controller design is to angoose certain agents as orientation agents (definitions will become clear in the context), for which some of the associated relative position vectors should achieve both desired distances and directions specified in the global coordinate frame. We note that a very general control framework for stabilizing an affine formation was recently proposed in Lin, Wang, Chen, Fu, and Han (2015), in which a strict assumption that the target formation should be globally rigid was imposed to generate a rigid shape with orientation constraint. Such an assumption is not required in the control strategy proposed in this paper. Also note that the formation orientation problem discussed in this paper is a stabilization control problem (i.e. to achieve a static formation with desired orientation), while a motion generation problem involving rigid formation orientation was discussed in Garcia de Marina, Jayawardhana, and Cao (2016) with a totally different control architecture.

Some preliminary results were presented in Park and Ahn (2015) and Sun and Anderson (2015). This paper extends the results reported in Park and Ahn (2015) and 
Sun and Anderson (2015), by providing a general and systematic approach to solve this control problem with a minimal number of orientation agents. Compared to Park and Ahn (2015) and Sun and Anderson (2015), the main extensions and contributions in this paper can be summarized as follows. First, the results to be discussed in this paper can be applied to stabilize rigid shapes and orientations without any restriction on agent numbers and ambient space dimensions, while Park and Ahn (2015) presented preliminary results focusing on proving asymptotic stability for a 2-D four-agent formation system. Second, we have removed the assumption that the target formation shapes are minimally rigid, which was a key assumption in Park and Ahn (2015) and Sun and Anderson (2015). Instead, by developing different approaches in the proofs, this paper only assumes that target formation shapes are infinitesimally rigid. Furthermore, by exploring several novel observations concerning the rigidity matrix from graph rigidity theory, we will also prove an exponential convergence to the desired formation shape with specified orientations. Note that the exponential stability renders the robustness property of the proposed formation control system in the presence small measurement errors or perturbations.

\subsection{Paper structure and notations}

The remaining parts of this paper are organized as follows. In Section 2, we introduce some background on graph and rigidity theory as well as the problem formulation. Certain novel results on graph rigidity theory will be shown in this section. Section 3 provides the main result. Typical simulation results are shown in Section 4. Finally, Section 5 concludes this paper. Proofs for some key lemmas are given in the Appendix.

Notations. The notations used in this paper are fairly standard. $\mathbb{R}^{n}$ denotes the $n$-dimensional Euclidean space. $\mathbb{R}^{m \times n}$ denotes the set of $m \times n$ real matrices. A matrix or vector transpose is denoted by a superscript $T$. The rank, image and null space of a matrix $M$ are denoted by $\operatorname{rank}(M), \operatorname{Im}(M)$ and $\operatorname{null}(M)$, respectively. We use $\operatorname{diag}\{x\}$ to denote a diagonal matrix with the entries of a vector $x$ on its diagonal, and $\operatorname{span}\left\{v_{1}, v_{2}, \cdots, v_{k}\right\}$ to denote the subspace spanned by a set of vectors $v_{1}, v_{2}, \cdots, v_{k}$. The symbol $I_{n}$ denotes the $n \times n$ identity matrix. Let $\mathbf{1}_{n}$ and $\mathbf{0}_{n}$ denote an $n$-tuple column vector of all ones and all zeros, respectively. When the subscripts are omitted, their dimensions should be clear in the context. The notations $\otimes$ and $\wedge$ represent the Kronecker product and cross product, respectively.

\section{Preliminaries and problem setup}

\subsection{Preliminary on graph theory}

Since formations of $n$ mobile agents are best described in terms of graph theory, we give a brief description of some of the basic definitions and facts needed. Consider an undirected graph with $m$ edges and $n$ vertices, denoted by $\mathcal{G}=(\mathcal{V}, \mathcal{E})$ with vertex set $\mathcal{V}=\{1,2, \cdots, n\}$ and edge set $\mathcal{E} \subset \mathcal{V} \times \mathcal{V}$. The neighbor set $\mathcal{N}_{i}$ of node $i$ is defined as $\mathcal{N}_{i}:=\{j \in \mathcal{V}:(i, j) \in \mathcal{E}\}$. The matrix relating the nodes to the edges is called the incidence matrix $H=\left\{h_{k i}\right\} \in$ $\mathbb{R}^{m \times n}$, whose entries are defined as (with arbitrary edge orientations for undirected formations considered here)

$$
h_{k i}=\left\{\begin{array}{cc}
1, & \text { the } k \text {-th edge sinks at node } i \\
-1, & \text { the } k \text {-th edge leaves node } i \\
0, & \text { otherwise }
\end{array}\right.
$$

For a connected and undirected graph, one has $\operatorname{rank}(H)=n-1$ and $\operatorname{null}(H)=\operatorname{span}\left\{\mathbf{1}_{n}\right\}$.

\subsection{Rigidity theory}

Given a vertex element $i \in \mathcal{V}$ we associate to it a point $p_{i}$ of Euclidean space $\mathbb{R}^{d}$. ${ }^{2}$ The column vector $p=$ $\left[p_{1}^{T}, p_{2}^{T}, \ldots, p_{n}^{T}\right]^{T}$ thus describes a framework $(\mathcal{G}, p)$ of $n$ agents, labelled by the set of vertices of $\mathcal{G}$. For any edge $k \in \mathcal{E}$ with head $j$ and tail $i$ which is consistent with the construction of the matrix $H$, consider the associated relative position vector defined as $z_{k}=p_{j}-p_{i}$. Let

$$
\begin{aligned}
z & =\left[z_{1}^{T}, z_{2}^{T}, \cdots, z_{m}^{T}\right]^{T} \in \mathbb{R}^{d m} \\
Z(z) & =\operatorname{diag}\left(z_{1}, z_{2}, \cdots, z_{m}\right) \in \mathbb{R}^{d m \times m}
\end{aligned}
$$

denote the associated column vector and block diagonal matrix, respectively. Note that there holds

$$
z=\left(H \otimes I_{d}\right) p
$$

With this notation at hand, we consider the smooth distance map

$r_{\mathcal{G}}: \mathbb{R}^{d n} \longrightarrow \mathbb{R}^{m}, r_{\mathcal{G}}(p)=\left(\left\|p_{i}-p_{j}\right\|^{2}\right)_{(i, j) \in \mathcal{E}}=Z(z)^{T} z$.

The rigidity of frameworks is then defined as follows.

Definition 1 (Asimow and Roth (1979)) A framework $(\mathcal{G}, p)$ is rigid in $\mathbb{R}^{d}$ if there exists a neighborhood $\mathbb{U}$ of $p$ such that $r_{\mathcal{G}}^{-1}\left(r_{\mathcal{G}}(p)\right) \cap \mathbb{U}=r_{\mathcal{K}}^{-1}\left(r_{\mathcal{K}}(p)\right) \cap \mathbb{U}$ where $\mathcal{K}$ is the complete graph with the same vertex set as $\mathcal{G}$.

2 In this paper we will focus on 2-D and 3-D formations, i.e. $d=2,3$. 
Two frameworks $(\mathcal{G}, p)$ and $(\mathcal{G}, \bar{p})$ are equivalent if $r_{\mathcal{G}}(p)=r_{\mathcal{G}}(\bar{p})$ and are congruent if $\left\|p_{i}-p_{j}\right\|=\left\|\bar{p}_{i}-\bar{p}_{j}\right\|$ for all $i, j \in \mathcal{V}$. A useful tool to study graph rigidity is the rigidity matrix, which is defined as the Jacobian matrix $R(p)=\frac{1}{2} \partial r_{\mathcal{G}}(p) / \partial(p)$. By inspection, $R(p)$ is an $m \times d n$ matrix given as

$$
R(p)=Z(z)^{T}\left(H \otimes I_{d}\right)
$$

Note that the entries of $R(p)$ only involve relative position vectors $z$, and we can rewrite it as $R(z)$. The rigidity matrix will be used to determine the infinitesimal rigidity of a framework, as shown in the following theorem.

Theorem 1 (Hendrickson (1992)) Consider a framework $(\mathcal{G}, p)$ in $d$-dimensional space with $n \geq d$ vertices and $m$ edges. It is infinitesimally rigid if and only if

$$
\operatorname{rank}(R(p))=d n-d(d+1) / 2
$$

Specifically, the framework $(\mathcal{G}, p)$ is infinitesimally rigid in $\mathbb{R}^{2}$ (resp. $\mathbb{R}^{3}$ ) if and only if $\operatorname{rank}(R(p))=2 n-3$ (resp. $\operatorname{rank}(R(p))=3 n-6)$. Obviously, in order to have an infinitesimally rigid framework, the graph should have at least $2 n-3($ resp. $3 n-6)$ edges in $\mathbb{R}^{2}\left(\right.$ resp. $\left.\mathbb{R}^{3}\right)$.

From Theorem 1, one knows that the dimension of the null space of $R(p)$ for an infinitesimally rigid framework $(\mathcal{G}, p)$ in the $d$-dimensional space is $d(d+1) / 2$. The following Lemma characterizes the structure of its null space.

Lemma 1 (Null space of the rigidity matrix) Suppose the framework $(\mathcal{G}, p)$ is infinitesimally rigid with the associated rigidity matrix denoted as $R(p)$.

- The $d=2$ case: The null space of $R(p)$ is of dimension 3 and is described as null $(R(p))=\operatorname{span}\left(q_{1}, q_{2}, q_{3}\right)$, where

$$
\begin{aligned}
& q_{1}=\mathbf{1}_{n} \otimes\left[\begin{array}{l}
1 \\
0
\end{array}\right] ; q_{2}=\mathbf{1}_{n} \otimes\left[\begin{array}{l}
0 \\
1
\end{array}\right] ; \\
& q_{3}=\left[\left(K_{3} p_{1}\right)^{T},\left(K_{3} p_{2}\right)^{T}, \cdots,\left(K_{3} p_{n}\right)^{T}\right]^{T},
\end{aligned}
$$

and the matrix $K_{3}$ is defined as

$$
K_{3}=\left[\begin{array}{cc}
0 & 1 \\
-1 & 0
\end{array}\right]
$$

- The $d=3$ case: The null space of $R(p)$ is of dimension 6 and is described as $\operatorname{null}(R(p))=$ $\operatorname{span}\left(q_{1}, q_{2}, q_{3}, q_{4}, q_{5}, q_{6}\right)$, where

$$
\begin{aligned}
& q_{1}=\mathbf{1}_{n} \otimes\left[\begin{array}{l}
1 \\
0 \\
0
\end{array}\right] ; q_{2}=\mathbf{1}_{n} \otimes\left[\begin{array}{l}
0 \\
1 \\
0
\end{array}\right] ; q_{3}=\mathbf{1}_{n} \otimes\left[\begin{array}{l}
0 \\
0 \\
1
\end{array}\right] ; \\
& q_{i}=\left[\left(K_{i} p_{1}\right)^{T},\left(K_{i} p_{2}\right)^{T}, \cdots,\left(K_{i} p_{n}\right)^{T}\right]^{T}, i=4,5,6 ;
\end{aligned}
$$

and the matrix $K_{i}$ is defined as

$$
K_{4}=\left[\begin{array}{ccc}
0 & 0 & 0 \\
0 & 0 & -1 \\
0 & 1 & 0
\end{array}\right] ; K_{5}=\left[\begin{array}{ccc}
0 & 0 & 1 \\
0 & 0 & 0 \\
-1 & 0 & 0
\end{array}\right] ; K_{6}=\left[\begin{array}{ccc}
0 & -1 & 0 \\
1 & 0 & 0 \\
0 & 0 & 0
\end{array}\right]
$$

The proof and detailed analysis on how to construct the above null vectors can be found in the appendix. In rigidity theory, any motion that lives in the null space of the rigidity matrix for an infinitesimally rigid framework is called an infinitesimal motion consisting of Euclidian motion (see e.g. Tay and Whiteley (1985), Anderson, Shames, Mao, and Fidan (2010)). The structure of the null space of a rigidity matrix was shown in e.g. (Zelazo, Franchi, Blthoff, and Robuffo Giordano, 2015, Theorem 2.16). The reason that we provide hereby an alternative proof is to show a unified and clearer structure of its null space and the corresponding translational motion and rotational motion. Such a clear structure on the null space analysis will be helpful and useful in the controller design and stability analysis, as will be shown in the main part of this paper.

The infinitesimal rigidity also guarantees the following property for a framework.

Lemma 2 Suppose the framework $(\mathcal{G}, p)$ is infinitesimally rigid. Then for any node $i$, the set of relative position vectors $p_{j}-p_{i}, j \in \mathcal{N}_{i}$ cannot all be collinear (in the 2-D case) or all be coplanar (in the 3-D case).

The proof can be found in the appendix. Lemma 2 will be useful for defining a feasible target formation by choosing some adjacent edges associated with certain agents (which will be discussed in Section 3.1).

\subsection{Gradient-based formation controller and problem formulation}

Let $d_{k_{i j}}$ denote the desired distance of edge $k$ which links agent $i$ and $j$. We further define

$$
e_{k_{i j}}=\left\|p_{i}-p_{j}\right\|^{2}-\left(d_{k_{i j}}\right)^{2}
$$

to denote the squared distance error for edge $k$. For ease of notation we may use $e_{k}$ and $d_{k}$ interchangeably in the sequel. This will also apply to $d_{k_{i j}}$ and $d_{k}, z_{k}$ and $z_{k_{i j}}$ 
in the following context when the dropping out of the dummy subscript $i j$ in each vector causes no confusion. if no confusion is expected. The squared distance error vector is denoted by $e=\left[e_{1}, e_{2}, \cdots, e_{m}\right]^{T}$. In this paper, we suppose that each agent is modeled by a single integrator $\dot{p}_{i}=u_{i}$ where $u_{i}$ is the controller to be designed for achieving the formation control objective.

In Krick et al. (2009), the following formation control system was proposed:

$$
\dot{p}_{i}=-\sum_{j \in \mathcal{N}_{i}}\left(\left\|p_{i}-p_{j}\right\|^{2}-d_{k_{i j}}^{2}\right)\left(p_{i}-p_{j}\right), i=1, \ldots, n
$$

The above control describes a steepest descent gradient flow of the following potential function

$$
V_{1}(p)=\frac{1}{4} \sum_{(i, j) \in \mathcal{E}}\left(\left\|p_{i}-p_{j}\right\|^{2}-d_{k_{i j}}^{2}\right)^{2}
$$

This potential function (7) for rigid shape stabilization and the associated gradient flow (6) have been extensively studied in the literature (see e.g. Krick et al. (2009); Cortés (2009); Dorfler and Francis (2010); Cao, Morse, Yu, Anderson, and Dasgupta (2011); Oh and Ahn (2014a), Anderson and Helmke (2014)). However, the above control and its extensions studied in these previous papers only stabilize a rigid formation shape, while the orientation of the formation is not specified. In this paper we will consider the problem of how to simultaneously stabilize a rigid shape and achieve a desired orientation for a target formation.

\section{Main result}

\subsection{Target formation and control framework}

Before describing the controller design, we first discuss how to define a target formation with the given interagent distance and formation orientation constraints. As mentioned in the above section, the commonly-used gradient-based controller (6) does not control the orientation and there are certain degrees of freedom relating to rotations for a converged formation. Intuitively, by regarding the rigid formation as a rigid body and specifying certain directions of some chosen edges in a global coordinate frame, the orientation of the overall rigid formation can be fixed. This will be the basic idea in the definition of a target formation and the controller design discussed in the sequel.

For simplifying the controller design and implementation, we choose one agent and a certain number of its neighboring agents as the specified agents to implement the additional orientation control task, with the associated edges between them being assigned with both distance constraints and orientation constraints. We term these agents with the additional orientation control task as orientation agents, and other agents as non-orientation agents. Thus, the target formation is defined with inter-agent distance constraints for all the agents, and orientation constraint for the chosen edges between orientation agents.

For the convenience of later analysis, we denote $\mathcal{G}_{0}$ as the underlying graph of the orientation control to distinguish it with the underlying graph $\mathcal{G}$ of the formation shape control. If the edge $(i, j)$ associated with agent $i$ and $j$ is chosen in the orientation control in $\mathcal{G}_{o}$, we denote it as $(i, j) \in \mathcal{E}_{o}$. The set of neighboring agents of orientation agent $i$ chosen in the orientation control is defined as $\mathcal{N}_{i}^{o}:=\left\{j \in \mathcal{V}:(i, j) \in \mathcal{E}_{o}\right\}$. The desired direction for the relative position vector $p_{j}-p_{i}$ for edge $(i, j) \in \mathcal{E}_{o}$ is denoted by a given vector $\hat{p}_{j i}:=\hat{p}_{j}-\hat{p}_{i}$. Thus, the orientation control is to additionally stabilize the relative position $p_{j}-p_{i}$ to the desired one $\hat{p}_{j}-\hat{p}_{i}$ with $(i, j) \in \mathcal{E}_{o}$. Due to the rigid body property of a desired rigid formation, the formation orientation can be determined by the directions of a certain set of desired relative position vectors. We show two examples, a 2-D four-agent rectangular formation and a 3-D tetrahedral formation depicted in Fig. 1 and Fig. 2, respectively, to illustrate the formation control framework.

Note that any two agents associated with one edge can be chosen as orientation agents, and there is no need to design a centralized algorithm for the selection of the orientation agents. To define a target formation with prescribed orientation, one can first choose one agent and then select one of its non-collinear relative vectors (for 2$\mathrm{D}$ formations) or two of its non-coplanar relative vectors (for 3-D formations) to specify the desired formation orientation. According to Lemma 2, such non-collinear or non-coplanar adjacent edges are guaranteed to exist for any agent to define a target formation. To sum up, we give a formal definition of a target formation.

Definition 2 (Target formation) The target formation is defined as $(\mathcal{G}, \tilde{p})$ which satisfies the following constraints

- Distance constraints: $\left\|\tilde{p}_{i}-\tilde{p}_{j}\right\|=d_{k_{i j}}, \forall(i, j) \in \mathcal{E}$;

- Orientation constraints: $\tilde{p}_{i}-\tilde{p}_{j}=\hat{p}_{i}-\hat{p}_{j}, \forall(i, j) \in \mathcal{E}_{o}$;

Note that there should hold $\left\|\left(\hat{p}_{j}-\hat{p}_{i}\right)\right\|=d_{k_{i j}}$ so that the orientation constraint is consistent with the formation shape constraint. In order to well define the orientation constraint, we need the following assumption.

Assumption $1 \mathrm{All}$ orientation agents should be equipped with coordinate systems with the same direction aligned with the global coordinate system.

Take the formation control formulation in Fig. 1 as an example. Since agents 1 and 2 are chosen as orienta- 


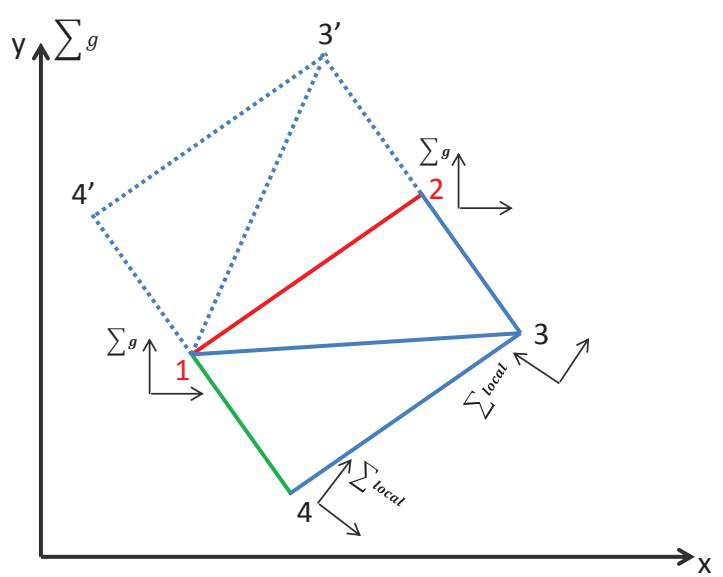

Fig. 1. An example of controlling a 2-D rigid formation with prescribed orientation. Agent 1 and one of its neighbors, agent 2 , are chosen as orientation agents. The relative position vector $p_{2}-p_{1}$ associated with edge $(1,2)$ is used to describe the desired orientation, which is denoted by red color (in this example $(1,2) \in \mathcal{E}_{o}$ ).

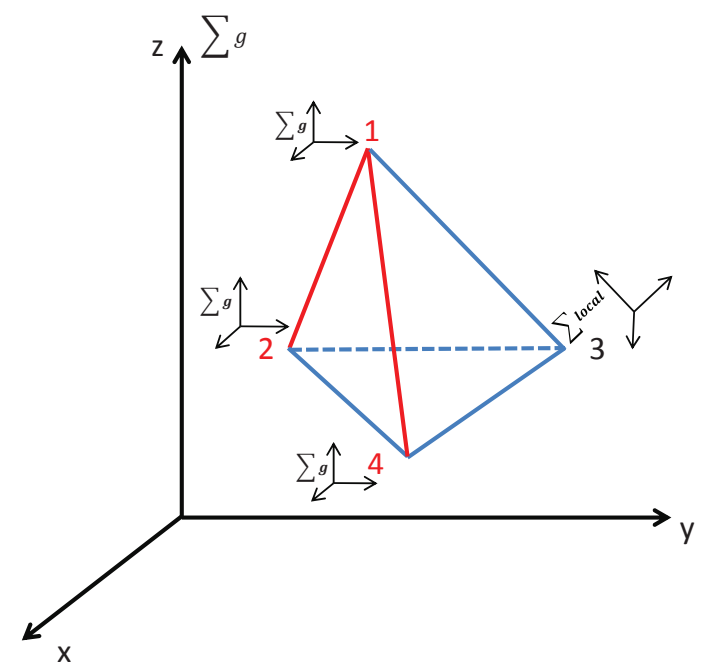

Fig. 2. An example of controlling a 3-D rigid formation with prescribed orientation. Agent 1 and two of its neighbors, agents 2 and 4 , are chosen as orientation agents. The relative position vectors $p_{2}-p_{1}$ and $p_{4}-p_{1}$ associated with edges $(1,2)$ and $(1,4)$ are used to describe the desired orientation, which are denoted by red color (in this example $(1,2),(1,4) \in \mathcal{E}_{o}$ ).

tion agents, their coordinate systems should be aligned with the global coordinate system denoted by $\sum_{g}$. Such a global coordinate system is required to define the desired relative position vector $\left(\hat{p}_{j}-\hat{p}_{i}\right)$ for $(i, j) \in \mathcal{E}_{o}$. Thus Assumption 1 provides a necessary condition for the controller design and implementation.

\subsection{Discussions on reflection ambiguity}

By specifying the direction of one edge in a 2-D formation, there exists a reflected formation with the same pre- scribed orientation (in the example in Fig. 1 the reflected formation, denoted by dotted blue lines, is obtained by the reflection via the mirror edge $(1,2))$. Such reflection ambiguity can be avoided by specifying the direction of an additional relative position vector (such as the one associated with edge $(1,4)$ ) or by assuming that the initial formation shape starts close to the desired one. In the latter case the formation shape will converge to the desired one instead of converging to the reflected one (by the convergence property of the gradient property of the proposed formation control system, to be proved in Theorem 2). Similarly, in the 3-D case there exists a reflected formation via the mirror plane spanned by that two chosen relative position vectors (in the example in Fig. 2 the mirror plane is spanned by that two relative vectors in edges $(1,2),(1,4))$. Such reflection ambiguity can be avoided by specifying the direction of an additional relative position vector (such as the one associated with edge $(1,3)$ ), or by assuming that the initial formation shape starts close to the desired one.

It might also be true that by setting the orientations for more edges may lead to a larger region of attraction, but this results in more orientation agents requiring the knowledge of the global coordinate system. Since in this paper we only focus on local convergence, the possibility of using more orientation edges will not be further exploited. In the current problem setting, the minimum number of orientation agents required in controller design is 2 (for 2-D rigid formations) and 3 (for 3 -D rigid formations). Such minimum number will be formally proved in later analysis which also guarantees the local convergence to a target formation with desired shape and orientation.

\subsection{Controller design}

We propose the following formation stabilization controller:

$$
\begin{aligned}
\dot{p}_{i}(t)= & \underbrace{\sum_{j \in \mathcal{N}_{i}}\left(p_{j}(t)-p_{i}(t)\right)\left(\left\|p_{j}(t)-p_{i}(t)\right\|^{2}-d_{k_{i j}}^{2}\right)}_{\text {shape control term, if }(i, j) \in \mathcal{E}} \\
& +\underbrace{\sum_{j \in \mathcal{N}_{i}^{o}}\left(\left(p_{j}(t)-p_{i}(t)\right)-\left(\hat{p}_{j}-\hat{p}_{i}\right)\right)}_{\text {orientation control term, if }(i, j) \in \mathcal{E}_{o}}
\end{aligned}
$$

It is obvious from Eq. (8) that the proposed control is distributed since only local information from neighboring agents in terms of relative positions is needed. In the later analysis we will also show that the overall system consisting of $n$ agents described by (8) is a gradient system associated with a cost function.

The above formation control system (8) can be written 
in a compact form

$$
\dot{p}=-R^{T} e-\left(L_{o} \otimes I_{d}\right) \bar{p}
$$

where $L_{o}$ is the Laplacian matrix of the underlying undirected graph $\mathcal{G}_{o}$ for the orientation control, and the vector $\bar{p}=\left[\bar{p}_{1}^{T}, \bar{p}_{2}^{T}, \cdots, \bar{p}_{n}^{T}\right]^{T}$ is defined as $\bar{p}_{i}=p_{i}-\hat{p}_{i}$ if $i$ is a chosen orientation agent ${ }^{3}$, or $\bar{p}_{i}=\mathbf{0}$ otherwise.

For the formation control system (9), the set of the desired equilibrium is described as

$\mathcal{M}=\left\{p \in \mathbb{R}^{d n} \mid e(p)=\mathbf{0}, p_{i}-p_{j}=\hat{p}_{i}-\hat{p}_{j}, \forall(i, j) \in \mathcal{E}_{o}\right\}$

which satisfies the constraints in Definition 2.

Example: We show an example to illustrate the above controller design. Suppose a group of four agents is tasked to achieve a rigid shape, with the additional orientation control assigned to edge $(1,2) \in \mathcal{E}_{O}$, which is illustrated in Fig. 1. The formation control system takes the following form

$$
\begin{aligned}
\dot{p}_{1}= & e_{12}\left(p_{2}-p_{1}\right)+e_{13}\left(p_{3}-p_{1}\right)+e_{14}\left(p_{4}-p_{1}\right) \\
& +\left(p_{2}-p_{1}\right)-\left(\hat{p}_{2}-\hat{p}_{1}\right) \\
\dot{p}_{2}= & e_{12}\left(p_{1}-p_{2}\right)+e_{23}\left(p_{3}-p_{2}\right)+\left(p_{1}-p_{2}\right)-\left(\hat{p}_{1}-\hat{p}_{2}\right) \\
\dot{p}_{3}= & e_{13}\left(p_{1}-p_{3}\right)+e_{23}\left(p_{2}-p_{3}\right)+e_{34}\left(p_{4}-p_{3}\right) \\
\dot{p}_{4}= & e_{14}\left(p_{1}-p_{4}\right)+e_{34}\left(p_{3}-p_{4}\right)
\end{aligned}
$$

(note that in the above equations the subscript notation for $e$ is slightly different to previous sections, in that $e_{i j}$ here denotes the squared distance error associated with the edge $(i, j) \in \mathcal{E})$. The Laplacian matrix $L_{o}$ for the underlying graph of orientation control is constructed as

$$
L_{o}=\left[\begin{array}{cccc}
1 & -1 & 0 & 0 \\
-1 & 1 & 0 & 0 \\
0 & 0 & 0 & 0 \\
0 & 0 & 0 & 0
\end{array}\right]
$$

and the vector $\bar{p}$ is constructed as $\bar{p}=\left[\left(p_{1}-\hat{p}_{1}\right)^{T},\left(p_{2}-\right.\right.$ $\left.\hat{p}_{2}^{T}, \mathbf{0}^{T}, \mathbf{0}^{T}\right]^{T}$. The formation system (11) can then be written in the compact form shown in (9).

\subsection{Properties of the formation control system}

In the following, we show several properties of the proposed control (8).

3 Note that the vector $\bar{p}_{i}$ is not an actual control input as $\hat{p}_{i}$ may not be available for agent $i$ (the actual control term is $\left.\hat{p}_{j i}:=\hat{p}_{j}-\hat{p}_{i}\right)$. The introduction of $\bar{p}_{i}$ is for the convenience of analysis and for writing a compact form of the formation system as in (9).
Lemma 3 The position of the formation centroid is preserved by the above control law (8).

Proof Denote by $p_{c} \in \mathbb{R}^{d}$ the center of the mass of the formation, i.e.,

$$
p_{c}=\frac{1}{n} \sum_{i=1}^{n} p_{i}=\frac{1}{n}\left(\mathbf{1}_{n} \otimes I_{d}\right)^{T} p
$$

One has

$$
\begin{aligned}
\dot{p}_{c}= & \frac{1}{n}\left(\mathbf{1}_{n} \otimes I_{d}\right)^{T} \dot{p} \\
= & -\frac{1}{n}\left(\mathbf{1}_{n} \otimes I_{d}\right)^{T}\left(R^{T} e+\left(L_{o} \otimes I_{d}\right) \bar{p}\right) \\
= & -\frac{1}{n}\left(\mathbf{1}_{n} \otimes I_{d}\right)^{T}\left(L_{o} \otimes I_{d}\right) \bar{p} \\
& -\frac{1}{n}\left(Z^{T}\left(H \otimes I_{d}\right)\left(\mathbf{1}_{n} \otimes I_{d}\right)\right)^{T} e
\end{aligned}
$$

Note that $\left(\mathbf{1}_{n} \otimes I_{d}\right)^{T}\left(L_{o} \otimes I_{d}\right) \bar{p}=\left(\left(\mathbf{1}_{n}^{T} L_{o}\right) \otimes I_{d}\right) \bar{p}=0$ and $\left(Z^{T}\left(H \otimes I_{d}\right)\left(\mathbf{1}_{n} \otimes I_{d}\right)\right)^{T} e=0$ because $\operatorname{null}(H)=$ $\operatorname{span}\left\{\mathbf{1}_{n}\right\}$. Thus $\dot{p}_{c}=0$, which indicates that the position of the formation centroid remains constant.

Lemma 4 For all non-orientation agents, their local coordinate systems are sufficient to implement the control law.

Proof Suppose agent $i$ is a non-orientation agent and its position in the global coordinate system $\sum_{g}$ is measured as $p_{i}^{g}$, while $p_{i}^{i}, p_{j}^{i}$ stand for agent $i$ and its neighboring agent $j$ 's positions, respectively, measured by agent $i$ 's local coordinate system. The controller for nonorientation agent $i$ can be written in its local coordinate as

$$
\dot{p}_{i}^{i}(t)=\sum_{j \in \mathcal{N}_{i}}\left(p_{j}^{i}(t)-p_{i}^{i}(t)\right)\left(\left\|p_{j}^{i}(t)-p_{i}^{i}(t)\right\|^{2}-d_{k_{i j}}^{2}\right)
$$

Clearly, there exist a rotation matrix $\mathcal{Q}_{i} \in \mathbb{R}^{d \times d}$ and a translation vector $\vartheta_{i} \in \mathbb{R}^{d}$, such that $p_{j}^{i}=\mathcal{Q}_{i} p_{j}^{g}+\vartheta_{i}$. We rewrite the controller (14) for the non-orientation agent $i$ in the global coordinate system $\sum_{g}$ as follows

$$
\begin{aligned}
\dot{p}_{i}^{g} & =u_{i}^{g}=\mathcal{Q}_{i}^{-1} u_{i} \\
& =\mathcal{Q}_{i}^{-1} \sum_{j \in \mathcal{N}_{i}} e_{k_{i j}}^{i}\left(p_{j}^{i}-p_{i}^{i}\right) \\
& =\sum_{j \in \mathcal{N}_{i}} e_{k_{i j}}^{g} \mathcal{Q}_{i}^{-1} \mathcal{Q}_{i}\left(p_{j}^{g}-p_{i}^{g}\right) \\
& =\sum_{j \in \mathcal{N}_{i}} e_{k_{i j}}^{g}\left(p_{j}^{g}-p_{i}^{g}\right)
\end{aligned}
$$


which has the same form as (14). Since $\mathcal{Q}_{i}$ and $\vartheta_{i}$ are chosen arbitrarily, the above equation indicates that the designed controllers for non-orientation agents are independent of the global coordinate basis.

To avoid notation complexity we omit the superscript in other parts of this paper for the convenience of analysis. This controller property has been illustrated in Fig. 1 and Fig. 2. In the example shown in Fig. 1, agent 3 or 4 is not an orientation agent and its coordinate system orientation does not need to be aligned with the global coordinate system. As a consequence of Lemma 4, the minimum number of orientation agents is 2 for a 2-D rigid formation and 3 for a 3-D rigid formation, which guarantees a minimum knowledge of global coordinate frame for the multi-agent formation group.

\subsection{Convergence analysis}

We will first show that the gradient property of the proposed controller and a general result on the convergence.

Theorem 2 The formation control system with the proposed controller (8) describes a gradient control system and the formation system converges to the largest invariant set in the set $\mathcal{O}(z)$ defined as

$$
\mathcal{O}(z)=\left\{z \mid R(z)^{T} e(z)+\left(L_{o} \otimes I_{d}\right) \bar{p}(z)=0\right\}
$$

Proof We choose the same potential function in (7) as the potential for the shape control, and the following potential function

$$
\begin{aligned}
V_{2} & =\frac{1}{2} \sum_{(i, j) \in \mathcal{E}_{o}}\left\|\left(p_{j}(t)-p_{i}(t)\right)-\left(\hat{p}_{j}-\hat{p}_{i}\right)\right\|^{2} \\
& =\frac{1}{2} \bar{p}^{T}\left(L_{o} \otimes I_{d}\right) \bar{p}
\end{aligned}
$$

for the orientation control. The composite potential function is then defined as $V=V_{1}+V_{2}$.

The dynamical system for the relative position $z$ defined in (1) is

$$
\begin{aligned}
\dot{z} & =\left(H \otimes I_{d}\right) \dot{p} \\
& =-\left(H \otimes I_{d}\right) R^{T} e-\left(H \otimes I_{d}\right)\left(L_{o} \otimes I_{d}\right) \bar{p}
\end{aligned}
$$

and the distance error system is described by

$$
\dot{e}=2 R \dot{p}=-2 R R^{T} e-2 R\left(L_{o} \otimes I_{d}\right) \bar{p}
$$

Note that the potential functions $V_{1}$ and $V_{2}$ are functions involving only relative position vectors in terms of $z$ and $e$ rather than the absolute position vector $p .4$ Thus, we can write the potential as $V(z)$ for the self-contained $z$ system (18). We then calculate the derivative of the potential $V_{1}$ and $V_{2}$ along the trajectories of system (18) and (19):

$$
\begin{aligned}
\dot{V}_{1} & =\frac{1}{2} e^{T} \dot{e}=e^{T}\left(-R R^{T} e-R\left(L_{o} \otimes I_{d}\right) \bar{p}\right) \\
& =-e^{T} R R^{T} e-e^{T} R\left(L_{o} \otimes I_{d}\right) \bar{p}
\end{aligned}
$$

and

$$
\begin{aligned}
\dot{V}_{2} & =\bar{p}^{T}\left(L_{o} \otimes I_{d}\right) \dot{\bar{p}} \\
& =\bar{p}^{T}\left(L_{o} \otimes I_{d}\right)\left(-R^{T} e-\left(L_{o} \otimes I_{d}\right) \bar{p}\right) \\
& =-\bar{p}^{T}\left(L_{o} \otimes I_{d}\right) R^{T} e-\bar{p}^{T}\left(L_{o} \otimes I_{d}\right)\left(L_{o} \otimes I_{d}\right) \bar{p}
\end{aligned}
$$

where in the second equality we have used the non-trivial result $\left(L_{o} \otimes I_{d}\right) \dot{\bar{p}}=\left(L_{o} \otimes I_{d}\right) \dot{p}$. The derivative of $V$ can be computed as

$$
\begin{aligned}
\dot{V}= & \dot{V}_{1}+\dot{V}_{2} \\
= & -e^{T} R R^{T} e-e^{T} R\left(L_{o} \otimes I_{d}\right) \bar{p} \\
& -\bar{p}^{T}\left(L_{o} \otimes I_{d}\right) R^{T} e-\bar{p}^{T}\left(L_{o} \otimes I_{d}\right)\left(L_{o} \otimes I_{d}\right) \bar{p} \\
= & -e^{T} R R^{T} e-2 e^{T} R\left(L_{o} \otimes I_{d}\right) \bar{p} \\
& -\bar{p}^{T}\left(L_{o} \otimes I_{d}\right)\left(L_{o} \otimes I_{d}\right) \bar{p} \\
= & -\left(R^{T} e+\left(L_{o} \otimes I_{d}\right) \bar{p}\right)^{T}\left(R^{T} e+\left(L_{o} \otimes I_{d}\right) \bar{p}\right) \\
\leq & 0
\end{aligned}
$$

The above derivative calculation thus implies that the formation system (8) describes a gradient descent flow for the composite potential $V$. Furthermore, the sublevel set of the potential $V(z)$ is compact with respect to the self-contained $z$ system (18). By LaSalle Invariance Principle, the solution of the formation system (18) converges to the largest invariant set in the set $\mathcal{O}(z)=$ $\{z \mid \dot{V}=0\}$ described in (16).

In general, a global picture of convergence analysis for a rigid formation control system is hard to obtain due to the existence of multiple equilibria (see discussions in e.g. Anderson and Helmke (2014)). Because the proposed control is a gradient law, the set $\mathcal{O}(z)$ also describes the set of equilibrium points for (8). Note that the desired equilibria set $\mathcal{M}$ is a subset of $\mathcal{O}(z)$. Similar to most works on rigid formation stabilization, in the following we will focus on local convergence analysis. In particular, we aim to show that the convergence to the target formation with desired distances and orientation is exponentially fast. The analysis is based on the linearization technique. We first compute the Jacobian of the vector field in the right-hand side of (9) around a

4 Also note that the distance error vector $e$ can be written in terms of $z$ according to the definition of $e$ in (5). 
desired equilibrium $\tilde{p} \in \mathcal{M}$ :

$$
\begin{aligned}
J_{f} & =\left.\frac{\partial\left(-R^{T} e-\left(L_{o} \otimes I_{d}\right) \bar{p}\right)}{\partial p}\right|_{p=\tilde{p}} \\
& =-\left.\frac{\partial R^{T}}{\partial p} e\right|_{p=\tilde{p}}-\left.R^{T} \frac{\partial e}{\partial p}\right|_{p=\tilde{p}}-\left.\frac{\partial\left(L_{o} \otimes I_{d}\right) \bar{p}}{\partial p}\right|_{p=\tilde{p}} \\
& =-\left.\left(R^{T} R+\left(L_{o} \otimes I_{d}\right)\right)\right|_{p=\tilde{p}}
\end{aligned}
$$

where $\left.\frac{\partial R^{T}}{\partial p} e(p)\right|_{p=\tilde{p}}=\mathbf{0}$ due to the fact that $e(p)=\mathbf{0}$ for a point $p$ in the equilibrium set $\mathcal{M}$, and $\left.\frac{\partial e}{\partial p}\right|_{p=\tilde{p}}=R$ according to the definition of the rigidity matrix.

Thus, the linearization equation of (9) is described as

$$
\delta \dot{p}=-\left(R^{T} R+L_{o} \otimes I_{d}\right) \delta p
$$

In the following, we prove that the convergence is exponentially fast.

Theorem 3 Suppose the target formation is infinitesimally rigid and initial positions of all the agents are chosen such that the initial formation is close to the desired formation. With the proposed control law (8), the convergence to the correct formation shape and orientation is exponentially fast.

Before giving the proof of the above result, we first show a key lemma on the property of the linearization matrix.

Lemma 5 Suppose the target formation is infinitesimally rigid and the orientation edges are selected according to Section 3.1. Then the linearization matrix $\mathcal{F}:=R^{T} R+L_{o} \otimes I_{d}$ is positive semidefinite and has $d$ zero eigenvalues. Furthermore, there holds $\operatorname{null}(\mathcal{F})=$ $\operatorname{null}\left(H \otimes I_{d}\right)=\operatorname{span}\left(\mathbf{1}_{n} \otimes I_{d}\right)$.

The proof can be found in the appendix.

Proof of Theorem 3 As shown in Lemma 3, the formation centroid is stationary. We construct an orthogonal matrix $Q \in \mathbb{R}^{d n \times d n}$ whose first $d$ rows are $\frac{1}{\sqrt{n}}\left(\mathbf{1}_{n} \otimes I_{d}\right)^{T}$. With $Q$, one can perform the coordinate transform on $p$ as

$$
\tilde{p}=Q p=\left[\begin{array}{l}
p^{o} \\
p_{r}
\end{array}\right]
$$

where $p^{o}=\sqrt{n} p_{c}$ according to the definition of $p_{c}$ in (12) and the structure of $Q$. From Lemma 3, one has $\dot{p}^{o}=\sqrt{n} \dot{p}_{c}=\mathbf{0}$. We also define a reduced transformation matrix $Q_{r} \in \mathbb{R}^{d(n-1) \times d n}$, obtained from $Q$ by removing the first $d$ rows. Note that there holds $p=Q^{-1} \tilde{p}=Q^{T} \tilde{p}$ and $p_{r}=Q_{r} p$. For the linearized system (24), one can obtain the following coordinate-transformed system

$$
\begin{gathered}
{\left[\begin{array}{c}
\delta \dot{p}^{o} \\
\delta \dot{p}_{r}
\end{array}\right]=Q \dot{\delta} p=-Q\left(R^{T} R+L_{o} \otimes I_{d}\right) \delta p} \\
:=-Q \mathcal{F} Q^{-1} \delta \tilde{p}
\end{gathered}
$$

According to the structure of the matrix $Q$, there holds

$$
\begin{aligned}
& Q \mathcal{F} Q^{-1}=\left[\begin{array}{c}
\frac{1}{\sqrt{n}}\left(\mathbf{1}_{n} \otimes I_{d}\right)^{T} \\
Q_{r}
\end{array}\right] \mathcal{F}\left[\frac{1}{\sqrt{n}}\left(\mathbf{1}_{n} \otimes I_{d}\right) \quad Q_{r}^{T}\right] \\
& =\left[\begin{array}{cc}
\mathbf{0} & \mathbf{0} \\
\mathbf{0} & Q_{r} \mathcal{F} Q_{r}^{T}
\end{array}\right]
\end{aligned}
$$

Therefore,

$$
\begin{aligned}
& \delta \dot{p}^{o}=\mathbf{0} \\
& \delta \dot{p}_{r}=-Q_{r} \mathcal{F} Q_{r}^{T} \delta p_{r}
\end{aligned}
$$

According to the definition of $Q_{r}$, the range space of $Q_{r}^{T}$ is the orthogonal complement of the subspace $\operatorname{span}\left(\mathbf{1}_{n} \otimes I_{d}\right)$. This, together with Lemma 5, implies that the linearization matrix $-Q_{r} \mathcal{F} Q_{r}^{T}$ is negative definite. Thus the convergence to the origin for the system (28) is locally exponentially fast. Since the system (28) is obtained from the system (24) by a linear coordinate transformation described in (25), the above statement also implies that the convergence to a point in the desired equilibrium $\mathcal{M}$ for the original system (9) is $l o-$ cally exponentially fast ((Khalil, 1996, Theorem 4.13)). For the linearized system, the guaranteed exponential convergence rate obtained in the linearization analysis is $\gamma=\lambda_{\min }\left(Q_{r} \mathcal{F} Q_{r}^{T}\right)$. Note that by the CourantFischer Theorem (Zhang, 2011, Theorem 8.9), the rate $\gamma=\lambda_{\min }\left(Q_{r} \mathcal{F} Q_{r}^{T}\right)$ is the same to the smallest positive eigenvalue of $\mathcal{F}$.

Remark 1 One may ask what happens if the formation is initially with a correct shape but needs to adjust the orientation by applying the designed controller (8). As can be seen from $(19), e(0)=0$ does not imply $\dot{e}(t)=0$ when the proposed controller (8) is applied. Thus, during the orientation adjustment the formation shape will be temporarily lost until the formation converges to the desired shape and orientation. If the formation shape should remain unchanged during the orientation adjustment, the control action should live in the null space of the rigidity matrix with a target formation shape derived in Lemma 1. A sufficient condition for the controller design in this case is to ensure that the $z$ system takes the form as $\dot{z}_{i}=\omega \times z_{i}$ (where $\omega$ indicates the angular velocity and $\times$ denotes the cross product) which guarantees a constant norm of $z$ and thus a preserved formation shape. 
Remark 2 In the above analysis we do not confine the formation to be minimally rigid (which is a commonly-used assumption in most literature on rigid formation control). Also we prove the local exponential convergence if the target formation is infinitesimally rigid (a more relaxed assumption than minimal rigidity). Exponential stability brings about several nice properties such as the robustness to system perturbations (e.g. measurement errors). This will be considered in future research, along the same research direction on robustness issues in rigid formation control Mou, Morse, Belabbas, Sun, and Anderson (2016). Note that the exponential convergence cannot be directly extended to the general convergence to a set stated in Theorem 2. This is because, as indicated in the proof of Theorem 3, the local exponential convergence to a target formation depends on the maximum rank condition of the rigidity matrix of a target formation, and for other formations defined in the set (16) one cannot guarantee that they are infinitesimally rigid.

\section{Illustrative examples}

In this section we provide several simulations to show formation behaviors and controller performance of the proposed control. Consider a 4-agent formation system, with the desired distances given as $d_{12}^{*}=d_{34}^{*}=3, d_{23}^{*}=$ $d_{14}^{*}=4, d_{13}^{*}=5$ corresponding to a rectangular shape. The initial positions for each agent are chosen as $p_{1}(0)=$ $[0,0]^{T}, p_{2}(0)=[-1,4]^{T}, p_{3}(0)=[5,3]^{T}$ and $p_{4}(0)=$ $[3,0]^{T}$, so that the initial formation shape is close to the target shape. When the conventional controller (6) is used, the trajectories of each agent and the final shape are depicted in Fig. 3, from which it can be seen that although the desired shape is achieved, the formation orientation is undefined.

We then consider the simulation using the proposed controller (8). We suppose the target formation should be the one with the rigid rectangular shape in addition that the relative position vector $p_{2}-p_{1}$ associated to edge $(1,2)$ should be aligned with the direction of the $y$-axis and the relative position vector $p_{4}-p_{1}$ associated with the edge $(1,4)$ should be aligned with the direction of the $x$-axis in the global coordinate. The desired relative vector for edge $(1,2)$ is set as $\hat{p}_{2}-\hat{p}_{1}=(0,3)^{T}$ and the initial positions are chosen as the same as the above simulation setting, which can avoid the reflected formation. The trajectories of each agent and the final shape are depicted in Fig. 4, which clearly show that the desired formation shape with the correct orientation is achieved and the formation centroid is preserved. The trajectories of each distance error and the orientation error for the edge $(1,2)$ are depicted in Fig. 5, which show an exponential convergence to the desired formation shape.

Lastly we show an example of stabilizing a rigid 3-D formation with desired orientation. The target forma-

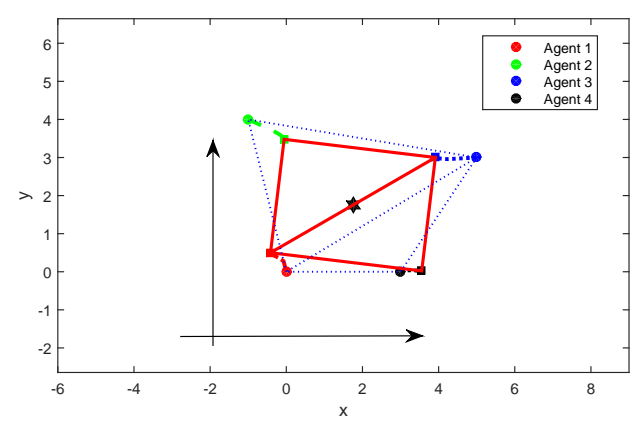

Fig. 3. Stabilization of a rigid rectangular formation without orientation control. The initial and final positions are denoted by circles and squares, respectively. The initial formation is denoted by dotted blue lines, and the final formation is denoted by red solid lines.

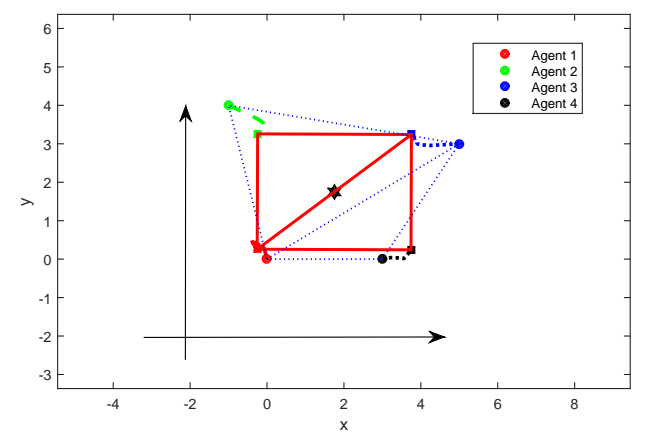

Fig. 4. Stabilization of a rigid rectangular formation with prescribed orientation. The initial and final positions are denoted by circles and squares, respectively. The initial formation is denoted by dotted blue lines, and the final formation is denoted by red solid lines. The black star denotes the formation centroid.

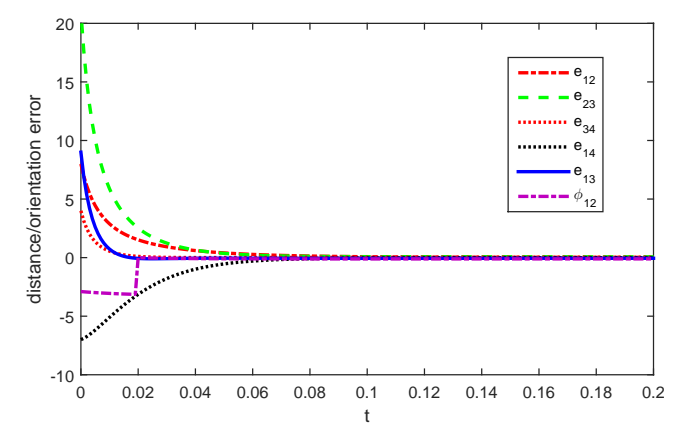

Fig. 5. Convergence of the distance/orentation errors with the proposed controller (8). The orientation error $\phi_{12}$ is defined as $\phi_{12}=\arctan \left(\left(p_{2, y}-p_{1, y}\right) /\left(p_{2, x}-p_{1, x}\right)\right)-\pi / 2$.

tion is a tetrahedron, with the desired distances given by $d_{12}^{*}=d_{13}^{*}=d_{14}^{*}=2, d_{23}^{*}=d_{34}^{*}=d_{24}^{*}=2 \sqrt{2}$. The desired orientation is that the edges $(1,3)$ and $(1,4)$ should be aligned with the the $x$-axis and the $z$-axis, respectively, which defines the orientation for the target tetrahedron formation. Following the control strategy in 


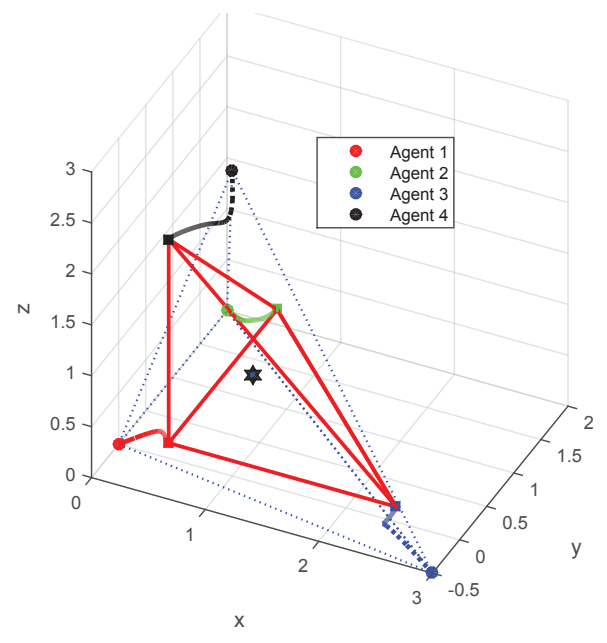

Fig. 6. Stabilization of a 3-D rigid formation with prescribed orientation. The initial and final positions are denoted by circles and squares, respectively. The initial formation is denoted by dotted blue lines, and the final formation is denoted by pink solid lines. The red line denotes the formation centroid.

Section 3 , the desired relative position vectors for edges $(1,3)$ and $(1,4)$ are defined as $\hat{p}_{1}-\hat{p}_{2}=(2,0,0)^{T}$ and $\hat{p}_{1}-\hat{p}_{4}=(0,0,2)^{T}$, in which agents 1,2 and 4 are chosen as orientation agents. The formation convergence is depicted in Fig. 6, which shows the successful achievement of the formation control task with both desired rigid shape and formation orientation.

\section{Conclusion}

In this paper we have discussed the formation control problem to achieve both desired rigid shapes and formation orientation. The designed controller combines the advantages of displacement-based approach and distance-based approach, by specifying a small number of agents as orientation agents which are tasked to control relative position vectors associated with them to desired directions. The proposed controllers are distributed in that only relative measurements from neighboring agents are required. For all non-orientation agents, any information about the global coordinate system is not required for them to implement the control, which guarantees a minimal requirement of the global knowledge of the global coordinate system. Certain simulation examples are provided to demonstrate the effectiveness of the proposed formation controllers.

\section{Acknowledgements}

The work of B. D. O. Anderson and Z. Sun was supported by NICTA, which is funded by the Australian Government through the ICT Centre of Excellence program, and by the Australian Research Council under grant DP130103610 and DP160104500. Z. Sun is also supported by the Prime Ministers Australia Asia Incoming Endeavour Postgraduate Award from Australian Government. The work of M.-C. Park and H.-S. Ahn was supported by the National Research Foundation of Korea (NRF) funded by the Ministry of Education, Science and Technology (NRF-2015M2A8A4049953).

\section{Appendix: proofs of several lemmas}

We first show a useful result on the dimension of the null space for two matrices and their product.

Lemma 6 Consider two matrices $A \in \mathbb{R}^{m \times n}$ and $B \in$ $\mathbb{R}^{n \times k}$ and the matrix product $C:=A B$. Then there holds $\operatorname{dim}(\operatorname{null}(C))=\operatorname{dim}(\operatorname{null}(B))+\operatorname{dim}((\operatorname{null}(A) \cap \operatorname{Im}(B))$.

Proof According to the Sylvester Rank Theorem (Zhang, 2011, Theorem 2.6), there holds $\operatorname{rank}(C)=$ $\operatorname{rank}(B)-\operatorname{dim}((\operatorname{null}(A) \cap \operatorname{Im}(B))$. Also, from the fundamental rank-nullity theorem one obtains $\operatorname{rank}(C)=$ $k-\operatorname{dim}(\operatorname{null}(C))$ and $\operatorname{rank}(B)=k-\operatorname{dim}(\operatorname{null}(B))$, which implies the desired result.

The following corollary is a consequence of Lemma 6 .

Corollary 1 Consider two matrices $A \in \mathbb{R}^{m \times n}$ and $B \in \mathbb{R}^{n \times k}$ and the matrix product $C:=A B$. If $\operatorname{dim}((\operatorname{null}(A) \cap \operatorname{Im}(B))=0$, then $\operatorname{null}(C)=\operatorname{null}(B)$.

Lemma 6 and Corollary 1 will be used later for analyzing the structure of null space of a rigidity matrix.

Proof of Lemma 1 The results can be verified by direct calculations. Here we aim to provide a full proof to show how to construct these null vectors, as the analysis will be useful in later proofs for some key lemmas.

From the assumption that the target formation is infinitesimally rigid, one has $\operatorname{rank}\left(\operatorname{null}\left(R^{T} R\right)\right)=$ $\operatorname{rank}(\operatorname{null}(R))=d(d+1) / 2$. The $d(d+1) / 2$ dimensional null space contains infinitesimal motions which preserve inter-agent distances, with dimension $d$ corresponding to the translational motion, and dimension $d(d-1) / 2$ corresponding to the rotational motion Hendrickson (1992). Also note that $R=Z^{T}\left(H \otimes I_{d}\right)$. Since the target formation is assumed to be infinitesimally rigid, the underlying graph for shape control should be at least connected, which implies that $\operatorname{null}\left(H \otimes I_{d}\right)=\operatorname{span}\left(\mathbf{1}_{n} \otimes I_{d}\right)$. Then according to Lemma 6 , there holds $\operatorname{null}\left(H \otimes I_{d}\right) \subset \operatorname{null}(R)$, which implies that $\operatorname{span}\left(\mathbf{1}_{n} \otimes I_{d}\right)$ is a $d$-dimensional subspace of the null space of $R$ corresponding to the translational motion. This proves that the null vectors $q_{1}, q_{2}$ are valid bases in the null space of $R$ for the 2-D formation case and $q_{1}, q_{2}, q_{3}$ are valid bases in the null space of $R$ for the 3 -D formation case.

We now divide the rest of the proof in the following two parts, according to the space dimensions: 
- The case of $d=2$ :

It is obvious that

$$
Q_{3}:=\left[\left(K_{3} z_{1}\right)^{T},\left(K_{3} z_{2}\right)^{T}, \cdots,\left(K_{3} z_{m}\right)^{T}\right]^{T}
$$

is a null vector of $Z^{T}$, i.e. $\operatorname{span}\left(Q_{3}\right) \subset \operatorname{null}\left(Z^{T}\right)=$ $\operatorname{null}\left(Z Z^{T}\right)$. Also note that

$$
\begin{aligned}
Q_{3} & =\left(I_{m} \otimes K_{3}\right) z=\left(I_{m} \otimes K_{3}\right)\left(H \otimes I_{d}\right) p \\
& =\left(H \otimes K_{3}\right) p=\left(H \otimes I_{d}\right)\left(I_{n} \otimes K_{3}\right) p
\end{aligned}
$$

which means that $\operatorname{span}\left(Q_{3}\right)$ is in the image of $\left(H \otimes I_{d}\right)$. Thus according to Lemma $6,\left(I_{n} \otimes K_{3}\right) p:=q_{3}$ is a null vector of the rigidity matrix $R$. Note that the null space of $R$ corresponding to the rotational invariance is of dimension 1, which implies that $q_{3}$ is the unique vector basis corresponding to the infinitesimal rotational motion.

- The case of $d=3$ :

It is obvious that the three vectors

$$
\begin{aligned}
Q_{4} & :=\left[\left(K_{4} z_{1}\right)^{T},\left(K_{4} z_{2}\right)^{T}, \cdots,\left(K_{4} z_{m}\right)^{T}\right]^{T} \\
& =\left(I_{m} \otimes K_{4}\right) z=\left(I_{m} \otimes K_{4}\right)\left(H \otimes I_{d}\right) p \\
Q_{5} & :=\left[\left(K_{5} z_{1}\right)^{T},\left(K_{5} z_{2}\right)^{T}, \cdots,\left(K_{5} z_{m}\right)^{T}\right]^{T} \\
& =\left(I_{m} \otimes K_{5}\right) z=\left(I_{m} \otimes K_{5}\right)\left(H \otimes I_{d}\right) p \\
Q_{6} & :=\left[\left(K_{6} z_{1}\right)^{T},\left(K_{6} z_{2}\right)^{T}, \cdots,\left(K_{6} z_{m}\right)^{T}\right]^{T} \\
& =\left(I_{m} \otimes K_{6}\right) z=\left(I_{m} \otimes K_{6}\right)\left(H \otimes I_{d}\right) p
\end{aligned}
$$

are linearly independent, and are three bases of null spaces of $Z^{T}$. Similarly to Eq. (29), $Q_{j}$ can be rewritten as

$$
Q_{j}=\left(H \otimes I_{d}\right)\left(I_{n} \otimes K_{j}\right) p
$$

for $j=4,5,6$, which implies that $\operatorname{span}\left(Q_{4}, Q_{5}, Q_{6}\right)$ is in the image of $\left(H \otimes I_{d}\right)$. Note that the null space of $R$ corresponding to the rotational invariance is of dimension $\frac{3 \times 2}{2}=3$. Thus according to Lemma 6 , the three vectors $\left(I_{n} \otimes K_{j}\right) p:=q_{j}$ for $j=4,5,6$ are the three null vector bases of $R$ corresponding to the infinitesimal rotational motion.

The proof is completed.

Proof of Lemma 2 The proof is done by trying to construct some null vectors of $R$ which are not in the null space spanned by the derived null vectors shown in Lemma 1, thus leading to contradictions to the rank condition of the infinitesimal rigidity in Theorem 1.

- The case of $d=2$ :

The infinitesimal rigidity excludes the case that $p_{j}-$ $p_{i}=\mathbf{0}$ for all $j \in \mathcal{N}_{i}$. Now we suppose that at least one $p_{j}-p_{i}$ is non-zero, and all the other $p_{k}-p_{i}$, $k \in \mathcal{N}_{i}$ can be described by a linear weight of $p_{j}-p_{i}$. Then construct a vector $\bar{q}=\left[\mathbf{0}^{T}, \mathbf{0}^{T}, \cdots,\left(K_{3}\left(p_{j}-\right.\right.\right.$ $\left.\left.\left.p_{i}\right)\right)^{T}, \cdots, \mathbf{0}^{T}\right]^{T}$ with the non-zero term $K_{3}\left(p_{j}-p_{i}\right)$ in the $2(i-1)+1$ to $2 i$ block. It is obvious that $R \bar{q}=0$ and $\bar{q} \notin \operatorname{span}\left(q_{1}, q_{2}, q_{3}\right)$. Thus, the existence of the null vector $\bar{q}$ in this case increases the dimension of the null space of $R$ and therefore $\operatorname{rank}(R)<2 n-3$, which violates the assumption that the framework is infinitesimally rigid. In conclusion, the set of relative position vectors $p_{j}-p_{i}, j \in \mathcal{N}_{i}$ cannot all be collinear for any $i$.

- The case of $d=3$ :

Similarly to the $d=2$ case, the infinitesimal rigidity excludes the case that all $p_{j}-p_{i}=\mathbf{0}$ for all $j \in \mathcal{N}_{i}$. The case that all $p_{j}-p_{i}, j \in \mathcal{N}_{i}$ are linearly dependent can be excluded by using the same argument as above. Now we suppose that two $p_{j}-p_{i}, p_{j^{\prime}}-p_{i}$, $j, j^{\prime} \in \mathcal{N}_{i}$ are non-zero, and all the other $p_{k}-p_{i}, k \in$ $\mathcal{N}_{i}$ can be described as linear combinations of these two. Then construct a vector $\bar{q}=\left[\mathbf{0}^{T}, \mathbf{0}^{T}, \cdots,\left(\left(p_{j}-\right.\right.\right.$ $\left.\left.\left.p_{i}\right) \wedge\left(p_{j^{\prime}}-p_{i}\right)\right)^{T}, \cdots, \mathbf{0}^{T}\right]^{T}$, with the non-zero term $\left(p_{j}-p_{i}\right) \wedge\left(p_{j^{\prime}}-p_{i}\right)$ in the $3(i-1)+1$ to $3 i$ block. By direct calculations, it can be shown that $R \bar{q}=0$ and $\bar{q} \notin \operatorname{span}\left(q_{1}, q_{2}, q_{3}, q_{4}, q_{5}, q_{6}\right)$. Thus, the existence of the null vector $\bar{q}$ in this case increases the dimension of the null space of $R$ and therefore $\operatorname{rank}(R)<3 n-6$, which violates the assumption that the framework is infinitesimally rigid. In conclusion, the set of relative position vectors $p_{j}-p_{i}, j \in \mathcal{N}_{i}$ cannot all be coplanar for any $i$.

By summarizing the above arguments, the proof is completed.

Proof of Lemma 5 First note that both $R^{T} R$ and $L_{o} \otimes$ $I_{d}$ are symmetric and positive semidefinite. From Lemma 1 one knows that $\operatorname{span}\left(\mathbf{1}_{n} \otimes I_{d}\right)$ is a subspace of the null space of $R$. Also, $\operatorname{span}\left(\mathbf{1}_{n} \otimes I_{d}\right)$ is a subspace of the null space of $L_{o} \otimes I_{d}$. Thus, there holds $\operatorname{span}\left(\mathbf{1}_{n} \otimes I_{d}\right) \subset$ $\operatorname{null}(\mathcal{F})$. We then show that there does not exist other null vectors in $\operatorname{null}(\mathcal{F})$.

We introduce a selection matrix, denoted by $J \in \mathbb{R}^{m \times m}$, whose $k$-th row is $e_{k}$ (i.e. the $k$-th standard basis) if the $k$-th edge is selected as the orientation edge, or the $k$-th row is an all-zero vector otherwise. Note that $J^{T}=J$. Denote the incidence matrix for the underlying graph of orientation control as $H_{o}$. By doing this, there holds $H_{o}=J H$ and thus $L_{o}=H_{o}^{T} H_{o}=H^{T} J J H$ since the underlying graph of orientation control is assumed to be undirected. Thus $\mathcal{F}=\left(H \otimes I_{d}\right)^{T} Z Z^{T}\left(H \otimes I_{d}\right)+(H \otimes$ $\left.I_{d}\right)^{T}\left(J J \otimes I_{d}\right)\left(H \otimes I_{d}\right)=\left(H \otimes I_{d}\right)^{T}\left(Z Z^{T}+J J \otimes I_{d}\right)(H \otimes$ $\left.I_{d}\right)$. We now divide the proof in the following two parts, according to the space dimensions:

- The case of $d=2$ :

From Lemma $1, Q_{3}$ is a null vector of $Z^{T}$ and $q_{3}$ is a null vector of $R$. By direct calculation, it holds $\left(Z Z^{T}+\right.$ $\left.J J \otimes I_{2}\right) Q_{3} \neq 0$, i.e. $Q_{3}$ is not a null vector of the matrix $\left(Z Z^{T}+J J \otimes I_{2}\right)$, which together with Corollary 1 implies that $q_{3}$ is not a null vector to the matrix $\mathcal{F}$. Thus, there holds null $(\mathcal{F})=\operatorname{span}\left(\mathbf{1} \otimes I_{2}\right)$, which 
implies that the null space of $\mathcal{F}$ is of dimension 2 and $\mathcal{F}$ has 2 zero eigenvalues.

- The case of $d=3$ :

We fix $j=4,5,6$. In this case, there are at least two non-zero rows in $J$, corresponding to at least two adjacent edges selected in the orientation control. From Lemma 1 , each $Q_{j}$ is a null vector of $Z^{T}$ and each $q_{j}$ is also a null vector of $R$. Following similar steps as above for the 2-D case and by direct calculation, it holds that $\left(Z Z^{T}+J J \otimes I_{3}\right) Q_{j} \neq 0$. Thus $Q_{j}$ are not null vectors of $\left(Z Z^{T}+J J \otimes I_{3}\right)$, which together with Corollary 1 implies $q_{j}$ are not null vectors of the matrix $\mathcal{F}$. Thus, there holds $\operatorname{null}(\mathcal{F})=\operatorname{span}\left(\mathbf{1} \otimes I_{3}\right)$, which implies that the null space of $\mathcal{F}$ is of dimension 3 and $\mathcal{F}$ has 3 zero eigenvalues.

By summarizing the above arguments, the statements in the lemma are proved.

\section{References}

Anderson, B. D., Shames, I., Mao, G., Fidan, B., 2010. Formal theory of noisy sensor network localization. SIAM Journal on Discrete Mathematics 24 (2), 684698.

Anderson, B. D. O., Helmke, U., 2014. Counting critical formations on a line. SIAM Journal on Control and Optimization 52 (1), 219-242.

Aranda, M., López-Nicolás, G., Sagüés, C., Zavlanos, M. M., 2015. Coordinate-free formation stabilization based on relative position measurements. Automatica 57, 11-20.

Asimow, L., Roth, B., 1979. The rigidity of graphs, II. Journal of Mathematical Analysis and Applications 68 (1), 171-190.

Cai, X., De Queiroz, M., 2015. Adaptive rigidity-based formation control for multirobotic vehicles with dynamics. IEEE Transactions on Control Systems Technology 23 (1), 389-396.

Cao, M., Morse, A. S., Yu, C., Anderson, B. D. O., Dasgupta, S., 2011. Maintaining a directed, triangular formation of mobile autonomous agents. Communications in Information and Systems 11 (1), 1-16.

Cortés, J., 2009. Global and robust formation-shape stabilization of relative sensing networks. Automatica 45 (12), 2754-2762.

Dorfler, F., Francis, B., 2010. Geometric analysis of the formation problem for autonomous robots. IEEE Transactions on Automatic Control 55 (10), 23792384.

Garcia de Marina, H., Jayawardhana, B., Cao, M., 2016. Distributed rotational and translational maneuvering of rigid formations and its applications. IEEE Transactions on Robotics. Accepted and in press. DOI: 10.1109/TRO.2016.2559511. Also vailabe at arXiv:1604.07849.

Hendrickson, B., 1992. Conditions for unique graph realizations. SIAM Journal on Computing 21 (1), 65-84.
Khalil, H. K., 1996. Nonlinear systems. Vol. 3. Prentice hall New Jersey.

Krick, L., Broucke, M. E., Francis, B. A., 2009. Stabilisation of infinitesimally rigid formations of multi-robot networks. International Journal of Control 82 (3), 423-439.

Lin, Z., Wang, L., Chen, Z., Fu, M., Han, Z., 2015. Necessary and sufficient graphical conditions for affine formation control. IEEE Transactions on Automatic Control, in press, 10.1109/TAC.2015.2504265.

Markdahl, J., Karayiannidis, Y., Hu, X., Kragic, D., 2012. Distributed cooperative object attitude manipulation. In: Proc. of IEEE International Conference on Robotics and Automation (ICRA). IEEE, pp. 29602965.

Meng, Z., Anderson, B. D. O., Hirche, S., 2016. Formation control with mismatched compasses. Automatica 69, 232-241.

Montijano, E., Zhou, D., Schwager, M., Sagues, C., June 2014. Distributed formation control without a global reference frame. In: Proc. of the 2014 American Control Conference. pp. 3862-3867.

Mou, S., Morse, A. S., Belabbas, M. A., Sun, Z., Anderson, B. D. O., 2016. Undirected rigid formations are problematic. IEEE Transactions on Automatic Control. Accepted and in press. DOI: 10.1109/TAC.2015.2504479.

Oh, K.-K., Ahn, H.-S., 2011. Formation control of mobile agents based on inter-agent distance dynamics. Automatica 47 (10), 2306-2312.

Oh, K.-K., Ahn, H.-S., 2014a. Distance-based undirected formations of single-integrator and double-integrator modeled agents in n-dimensional space. International Journal of Robust and Nonlinear Control 24 (12), 1809-1820.

Oh, K.-K., Ahn, H.-S., Feb 2014b. Formation control and network localization via orientation alignment. IEEE Transactions on Automatic Control 59 (2), 540-545.

Oh, K.-K., Park, M.-C., Ahn, H.-S., 2015. A survey of multi-agent formation control. Automatica 53, 424 440.

Pais, D., Cao, M., Leonard, N. E., 2009. Formation shape and orientation control using projected collinear tensegrity structures. In: Proc. of the 2009 American Control Conference. IEEE, pp. 610-615.

Park, M.-C., Ahn, H.-S., 2015. Distance-based control of formations with orientation control. In: Proc. of the 54th IEEE Conference on Decision and Control. pp. 2199-2104.

Ren, W., Beard, R. W., 2008. Distributed consensus in multi-vehicle cooperative control. Springer.

Sun, Z., Anderson, B. D. O., 2015. Rigid formation control with prescribed orientation. In: Proc. of the IEEE Multi-Conference on Systems and Control (MSC15). pp. 639-645.

Sun, Z., Mou, S., Anderson, B. D. O., Morse, A. S., 2014. Formation movements in minimally rigid formation control with mismatched mutual distances. In: Proc. of the 53rd Conference on Decision and Control. IEEE, 
pp. $6161-6166$.

Tay, T.-S., Whiteley, W., 1985. Generating isostatic frameworks. Structural Topology 1985 11, 21-49.

Tian, Y.-P., Wang, Q., 2013. Global stabilization of rigid formations in the plane. Automatica 49 (5), 14361441.

Wang, L., Markdahl, J., Hu, X., 2011. Distributed attitude control of multi-agent formations. In: Proc. of the 18th IFAC World Congress. pp. 2965-2971.

Xiao, F., Wang, L., Chen, J., Gao, Y., 2009. Finite-time formation control for multi-agent systems. Automatica 45 (11), 2605-2611.

Zelazo, D., Franchi, A., Blthoff, H. H., Robuffo Giordano, P., 2015. Decentralized rigidity maintenance control with range measurements for multi-robot systems. The International Journal of Robotics Research 34 (1), 105-128.

Zhang, F., 2011. Matrix theory: basic results and techniques. Springer Science \& Business Media.

Zhao, S., Zelazo, D., 2016. Bearing rigidity and almost global bearing-only formation stabilization. IEEE Transactions on Automatic Control 61 (5), 1255-1268. 